\title{
DYNAMIC MODELLING OF RETORT THERMODYNAMICS OF OIL SHALES
}

\author{
D. R. VAN PUYVELDE* \\ Granular Flow Research \\ 78 Degraves Crescent \\ Wanniassa ACT 2903 \\ Canberra, Australia
}

Oil shale has been a source of oil for many decades in several countries. Globally the resurgence of oil shale is a result from: rising oil prices, which has made oil production from oil shale economic; countries securing their domestic energy supply, which reduces their reliance on oil imports from politically unstable states; and increasing demand for conventional oil, which is not being met by new conventional oil discoveries.

Production of oil from oil shale requires the organic material in oil shale to be retorted. This usually takes place through contacting dried oil shale with hot recycled shale ash. This paper presents a detailed model of heat transfer in granular material and combines this with oil shale thermodynamics to estimate the rate of oil production from oil shale. A range of scenarios are presented including changing oil shale composition, adjusting the particle size, changing the initial temperature of the oil shale and adjusting the extent of mixing between the two materials.

It is shown that chemical reactions of oil shale have a significant influence on the final equilibrium temperature and the time taken to reach this temperature. This equilibrium temperature also depended on the initial temperature of the materials and the ratio between those materials but was not influenced by the particle size nor the extent of mixing of the mixture. However, the time taken to reach this equilibrium temperature was affected by all variables.

\section{Introduction}

The demand for primary energy sources continues to grow and the International Energy Agency [1] predicts that this demand will more than double between 1990 and 2030. The majority of this growth is expected to come from fossil fuels. The increase in demand combined with the fear of peak oil

\footnotetext{
*Corresponding author: e-mail d_van_puyvelde@optusnet.com.au
} 
and higher oil prices are making alternative fuel sources more promising in the short to medium term. This means that previously uneconomical oil reserves are being exploited, and it also creates the opportunity for oil shales and tar sands to become an economic commodity in the oil production industry. Some countries, such as Brazil, Estonia and China, have already started exploiting their oil shale deposits on a commercial level, but this is more a function of domestic energy security rather than reflecting a global demand for oil shale products. Nevertheless, the development of unconventional oil deposits will be required to meet the growing global energy demands.

Oil shales contain kerogen - an organic material - that can be removed using retorting technologies and reformed into fuels and petrochemicals. In order to extract this kerogen, oil shale needs to be heated, in most cases to around $500{ }^{\circ} \mathrm{C}$ but this depends on the oil shale. There are a number of technologies that can be used to extract oil from oil shale. This can be either done in-situ - as is being trialled at Green River, or $e x$-situ - as used in the Estonian oil shale industry. The focus of this paper is on ex-situ processing.

Typically ex-situ processing involves the mining of oil shale, transporting this oil shale to the processing plant and crushing the oil shale to an acceptable particle size that can be processed. The ideal particle size depends on the type of oil shale and process, but generally fine particles (say less than $5 \mathrm{~mm}$ ) are difficult to handle. The oil shale is then dried in a preheater, and the dried oil shale passed to a retort. Within the retort, the oil shale is mixed with recycled hot shale ash (produced from the next step of the process) and the heat transfer from the hot shale ash heats up the oil shale to drive the retorting reactions. The product from the retort can be reformed in hydrotreating plants, and is the base product for oil production from the oil shale. The amount of kerogen and type of deposit influence the operating conditions, such as the final processing temperature and the ratio of materials required. Since this is predominantly the problem of heat transfer in a granular material, the discrete nature of particles is important to consider. The kerogen that is retorted in this part of the process passes through the bed of solids. Some of this gas then reacts with the particles and forms a carbon layer on the surface of the particles of what is now the shale ash. This layer of carbon is combusted to raise the temperature of the shale ash, which is then recycled to other parts of the plant to drive the drying and retorting reactions. Schematically this process is shown in Fig. 1.

There are three commercial processes for producing oil from oil shale [2]. The Galoter and Alberta Taciuk processes both use rotary kilns to contain the retorting reaction. The retorting of oil shale is driven by granular heat transfer between oil shale and recycled shale ash since shale ash is used as heat carrier. The Galoter process uses separate process units for shale preheating and combustion while the Alberta Taciuk processor integrates all these processes into a single operational unit. The Kiviter process is slightly different in that the retorting is driven by heat transfer from a hot inert gas to 
a packed bed of particles. This process only accepts large particle sizes. The different processes produce different oil yields and compositions that reflect the different heating rates of oil shale.

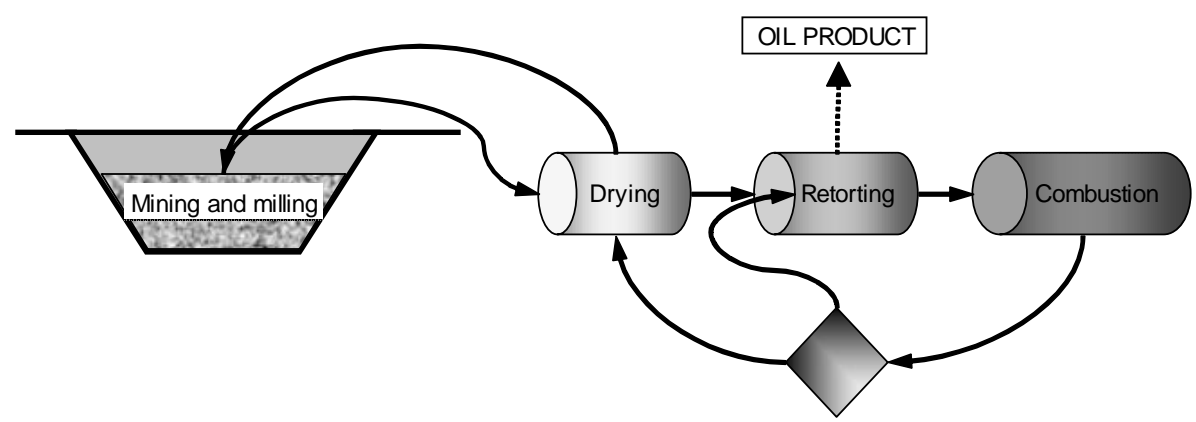

Fig. 1. Schematic of a typical oil shale process.

The purpose of this paper is to describe a model that has been developed which estimates the retorting processes in the retort zone of the Galoter or Alberta Taciuk processes. A thermodynamic model of oil shale is presented, and this is combined with a generic heat transfer model between solid particles. The resulting model can be used to determine the rate of heating of oil shale particles. In turn this can be used to estimate the rate of oil production for a set of specified process conditions and oil shale types.

\section{Thermal characteristics of oil shale and shale ash}

Overall, oil shale retorting reactions are exothermic and involve drying, mineral reactions and kerogen retorting reactions. All these reactions occur over a wide range of temperatures and depend on the oil shale composition. Berkovich et al [3-5] developed a thermodynamic model to estimate the enthalpy of oil shales during heating. This model was based on the individual components of Australian oil shales and was tested extensively against other oil shale samples. Thermal characterisation for other oil shales was also carried out by Kok et al [6]. Integrating the model by Berkovich [5] over a temperature range gives a unique profile for the oil shale, as shown in Fig. 2.

The model can be adjusted to estimate the thermal profile of other oil shales by adjusting the composition of oil shale in the thermodynamic model. As shown in Fig. 2, the profile consists of two components, one being the sensible heat of heating oil shale, and the other being the heat of reaction from the irreversible reactions. The characteristics and modelling parameters of the oil shale used in this paper are given in Table 1. 


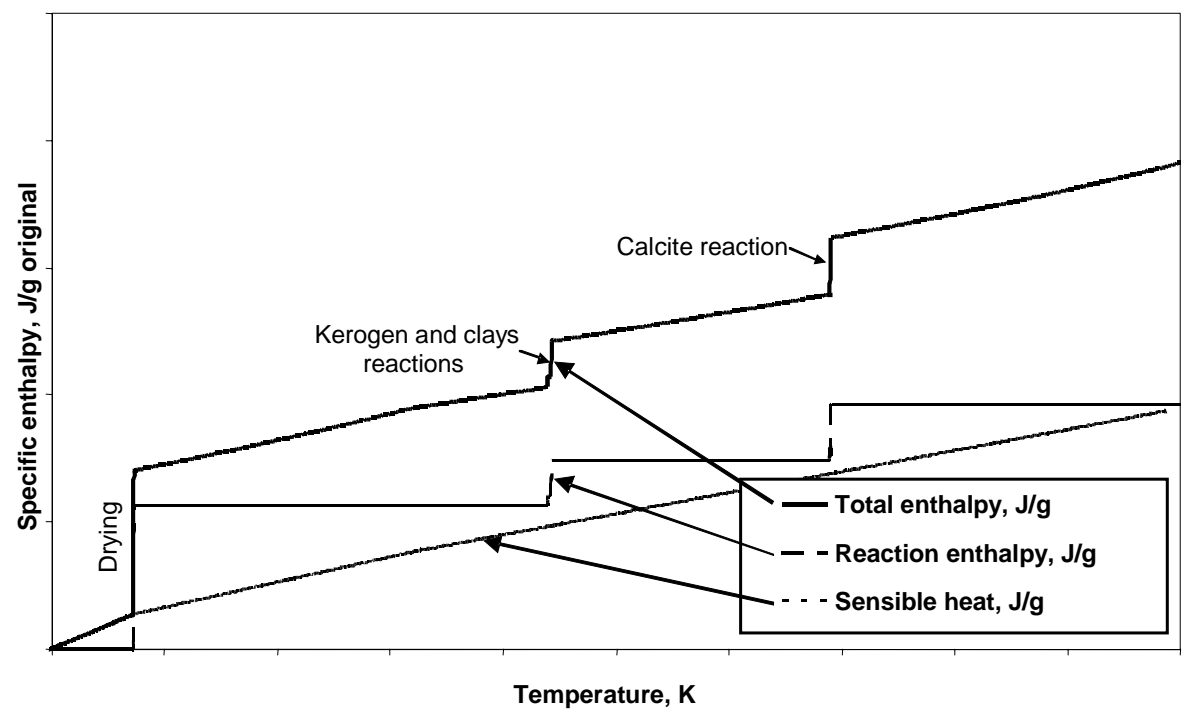

Fig. 2. Thermal profile of an oil shale, showing drying, retorting reactions and mineral reactions.

Table 1. Characteristics and modelling parameters of the oil shale used in this paper

\begin{tabular}{|c|c|c|c|c|c|c|}
\hline \multirow[t]{2}{*}{ Component } & \multirow{2}{*}{$\begin{array}{l}\text { Composi- } \\
\text { tion, } \\
\text { mass } \%\end{array}$} & \multicolumn{2}{|c|}{ Experimental } & \multicolumn{3}{|c|}{ Modelling } \\
\hline & & $\begin{array}{c}\text { Reaction } \\
\text { temperature, } \\
\mathrm{K}\end{array}$ & $\begin{array}{l}\text { Reaction } \\
\text { enthalpy, } \\
\text { J/g }\end{array}$ & \begin{tabular}{|} 
Reaction \\
temperature, \\
$\mathrm{K}$
\end{tabular} & $\begin{array}{c}\text { Initial reaction } \\
\text { temperature, } \\
\mathrm{K}\end{array}$ & $\begin{array}{c}\text { Decay } \\
\text { constant }\end{array}$ \\
\hline Water & 25 & 373 & 564.75 & 40 & 353 & 17.37 \\
\hline Siderite & 7 & 740 & 40.94 & 10 & 740 & 4.34 \\
\hline $\begin{array}{l}\text { Kerogen, } \\
\text { kaolonite } \\
\text { and illite }\end{array}$ & 55 & 743 & 136.80 & 50 & 718 & 21.71 \\
\hline Calcite & 13 & 99 & 220.75 & 50 & 990 & 21.71 \\
\hline
\end{tabular}

\section{Mathematical modelling of oil shale thermal behaviour}

Mathematically, estimating the temperature and composition of oil shale simultaneously is difficult, especially in the reaction regions where heat exchange does not translate to a change in temperature. The above profiles were approximated so that they would be easily programmable.

The basic assumption is that the overall thermal response of oil shale was approximated by adding thermal characteristics of each component while the material is being heated. The response for each component was approximated by an exponential decay function, which equaled zero before a certain threshold temperature and then increased according to an exponential decay function. A reaction temperature range was assumed in this approximation 
and a decay constant was taken as a function of this temperature range. The enthalpy change, $\Delta H_{r x n, n}$, from each component, $n$, is given by:

$$
\Delta H_{r x n, n}(T)=\left\{\begin{array}{l}
0 \text { if } T_{n, i}<T \\
\Delta \bar{H}_{r x n, n}\left(1-e^{-\frac{\left(T-T_{n, i}\right)}{k}}\right) \text { if } T_{n, i} \geq T
\end{array}\right.
$$

where $T_{n, i}$ is the initial reaction temperature, $T$ is the current temperature of the oil shale particle, $\Delta H_{r x n, n}$ is the reaction enthalpy ( $\mathrm{J} / \mathrm{g}$ of original material) and $k$ is the decay constant. Figure 3 shows how this approximation is carried out.

The sensible heat, $\Delta H_{\text {sens, oilshale }}(T)$, for this oil shale was estimated as:

$$
\Delta H_{\text {sens, oil shale }}(T)=\left\{\begin{array}{l}
0 \text { if } T<298 \mathrm{~K} \\
0+0.54 T \text { if } T \geq 298 \mathrm{~K} \text { and } T<373 \mathrm{~K} \\
75.54+1.01 T \text { if } T \geq 373 \mathrm{~K} \text { and } T<623 \mathrm{~K} \\
251.01+1.21 T \text { if } T \geq 623 \mathrm{~K}
\end{array}\right.
$$

The total enthalpy of oil shale is estimated as the sum of reaction enthalpies of the components and the sensible heat. Thus:

$$
\Delta H(T)=\Delta H_{\text {sens, oil shale }}(T)+\sum_{n} \Delta H_{r x n, n}(T)
$$

for all $n$ components in the oil shale.

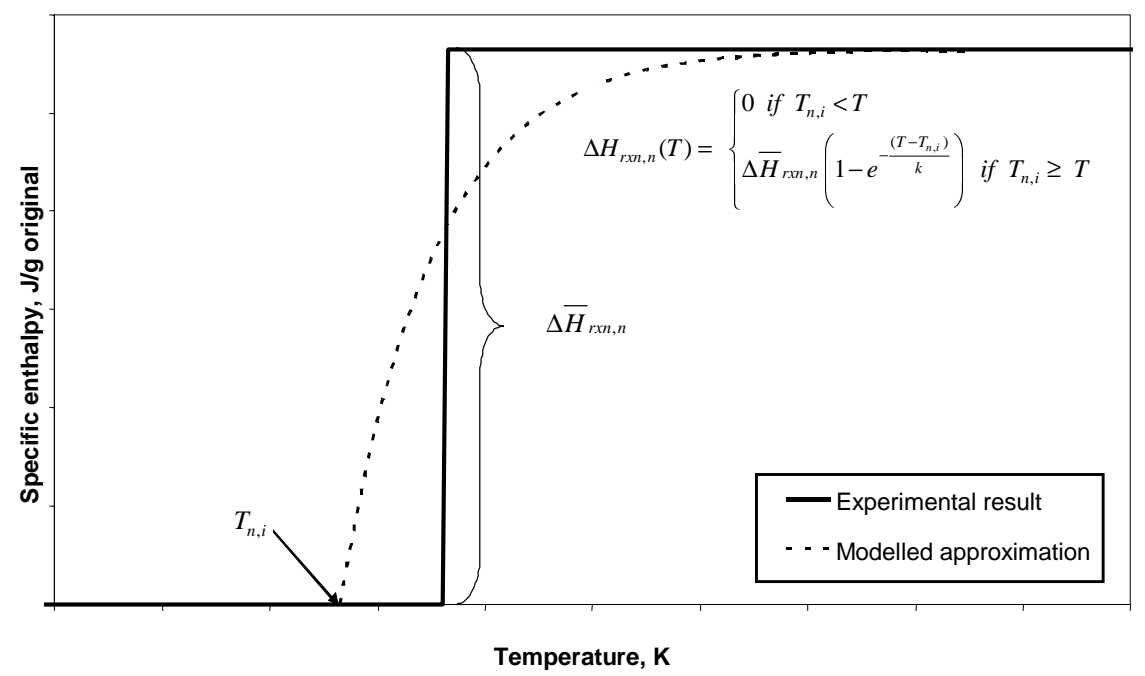

Fig. 3. Step function approximated by exponential decay. 


\section{Mathematical modelling of oil shale ash thermal behaviour}

Shale ash also has a unique thermal profile. However, this is simpler compared to the thermal profile of oil shale. It can be readily assumed that the oil shale reactions are irreversible. Hence only a sensible heat component is required to estimate the enthalpy content of shale ash. The thermal profile of shale ash equals the sensible heat rate of oil shale at the highest temperature. The enthalpy of shale ash as a function of temperature is given by:

$$
\Delta H_{\text {sens,shale ash }}(T)=\left\{\begin{array}{l}
0 \text { if } T<298 \mathrm{~K} \\
1.21 T \text { if } T \geq 298 \mathrm{~K}
\end{array}\right.
$$

The resulting model, based on Berkovich's experimental observations, can be used to simultaneously calculate the enthalpy content and temperature of an oil shale sample. It should be noted that the experimental work did not test the effects of heating rate on the quality of the oil product from the retort. Even though there have been studies describing some of these effects, they will not be considered in this paper. Further work on the complex kerogen retorting chemistry can be incorporated in future versions of the model. To illustrate the granular material heat transfer model, a generic oil shale sample is used. This is based on the range of components in Berkovich et al [4], and is shown in Table 1.

\section{Granular medium heat transfer model}

Heat transfer within particles, between particles or between particles and reactor walls (or heat transfer tubes) has been studied in quite some detail over the years. However, most of the experimental work and subsequent modelling work have focussed on quite specific process conditions (e.g. ignition of coal particles or heat transfer between sand and metal tubes). Moreover, these models are generally limited to steady state heat transfer with a predominant mode of heat transfer (i.e. radiation or conduction or convection).

Oil shale processing covers a wide temperature range and includes all of the above modes of heat transfer. Most of the heat transfer studies involving oil shale have been focussed on the fundamental chemistry of the oil shales, and while these are important, further modelling work needs to be done for process design.

This paper will not provide a detailed overview of the heat transfer literature. Instead the focus is on developing a dynamic heat transfer model that builds on the approach by Yagi \& Kunii [7] and Kunii \& Smith [8] and the thermodynamic model above.

Figure 4 illustrates the heat transfer paths between two particles based on the model by Yagi \& Kunii [7]. The particles are assumed to be spherical. 
Heat transfer path 1 is the conductive heat transfer between the particles through the points of contact between the particles. Path 2 is the heat transfer by conduction and convection through the fluid film near the place of contact. Heat transfer path 3 is the net radiation heat transfer from the surface of a hot particle to the surface of cold particle. Radiation from the hot particle to the void space between particles and radiation from the void space to the cold particle are described as paths 4 and 5, respectively. Lastly path 6 describes the heat transfer to/from the surface of the particles into the bulk of those particles due to conduction. The total heat transfer rate through all six paths, $Q$, equals: $Q=U A \Delta T$, where $U$ is the overall heat transfer coefficient, $A$ is the heat transfer area of the particle and $\Delta T$ is the temperature difference between the particles.

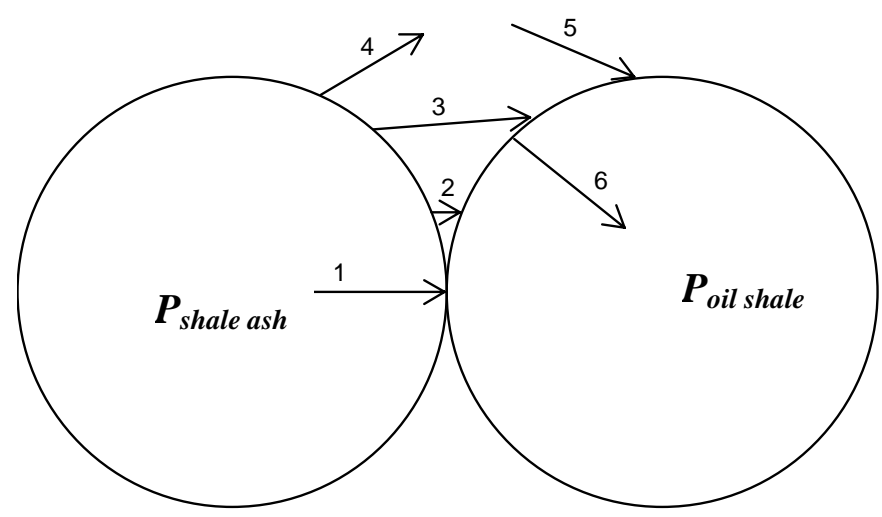

Fig. 4. Heat transfer paths between particles [7].

The following assumptions are made in the current model:

1. Conduction between the particles is negligible. This is justified since the area at the points of contact between hard particles is very small compared to the surface area of the particles, and hence the surface area for conduction is small.

2. Conduction into the particles is not a rate limiting step in heat transfer.

3. The gas produced from the retort reaction flow slowly past the particles so that they do not produce forced convection over the particle surfaces.

4. All void spaces are at a uniform temperature, and this temperature equals the surface area averaged temperature of the material surrounding the voids. It is assumed that enthalpy content of the void spaces is negligible compared to that of the particles. The surface area average temperature is used since surface area is more important to heat transfer rates than the mass (total enthalpy) of the particle. 
5. Only heat transfer from the hot particles to the cold particles is calculated. This implies that all oil shale particles are at a uniform temperature, while all shale ash particles are also at a different uniform temperature.

Thus the remaining heat transfer paths in this model are natural convection from the gas in the void spaces to the particles, and radiation between particles.

\section{Natural convective heat transfer}

To determine the natural convective heat transfer between the gas and the particles, the heat transfer coefficient is calculated by using the Nusselt, $\overline{N u \tilde{L}}$, number [9]:

$$
\overline{N u \tilde{L}}=\frac{\bar{h} \cdot \tilde{L}}{k}
$$

where $\bar{h}$ is the heat transfer coefficient from the solid surface, $\tilde{L}$ is the characteristic length of the particle, which for a sphere is $\pi \mathrm{D} / 2$ and $k$ is the gaseous phase thermal conductivity. $\overline{N u \tilde{L}}$ can be calculated using: $\overline{N u \tilde{L}}=\left(a+0.331 b \cdot R a \tilde{L}^{1 / 6}\right)^{2}$, where $a$ is 1.77 for spheres, $b$ equals $1.17 /\left[1+(0.5 / \operatorname{Pr})^{9 / 16}\right]^{8 / 27}$, Ra $\tilde{L}$ is the Rayleigh number and Pr is the Prandtl

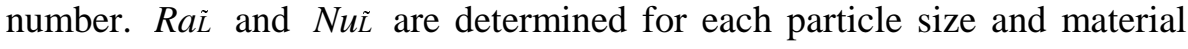
type. The heat transfer coefficients differ for different particle sizes and temperature differences.

The convective heat transfer rates to or from the different material, $Q_{n c, i}$, are calculated using: $Q_{n c, i}=\bar{h}_{i} A_{i}\left(T_{\text {void }}-T_{i}\right)$, where the subscript $i$ denotes either oil shale or shale ash, $A_{i}$ is the heat transfer area taken as the total surface area for material $i, T_{\text {void }}$ is the mean gas temperature and $T_{i}$ is the average particle temperature of material $i$.

Since this is an unsteady state model, the convective heat transfer, $q_{n c, i}$, during a specified time period, $\Delta t$, is calculated for each material type using:

$$
q_{n c, i}=Q_{n c, i} \cdot \Delta t,
$$

where the time step is estimated to achieve a smooth dynamic response.

To ensure conservation of energy, each $q_{n c, i}$ is scaled to the lowest absolute value of $q_{n c, i}$. In reality, if the convective heat transfer gain estimate for oil shale was larger than the heat lost for shale ash, the gases in the void space would cool. However, this would require an energy accumulation term and a better understanding of the gas phase, which is beyond the scope of this modelling exercise. 


\section{Radiation heat transfer}

The radiation heat transfer is important due to the high temperatures and the large particle sizes used in the oil shale processors [10]. Radiation heat transfer occurs from the hot material, the shale ash, to the colder material, the oil shale.

The radiation heat transfer rate, $Q_{r}$, between the materials is determined as:

$$
Q_{r}=\sigma \cdot \mathcal{E} \cdot A_{\text {Rad }} \cdot M \cdot\left(T_{\text {ash }}{ }^{4}-T_{\text {shale }}{ }^{4}\right),
$$

where $\sigma$ is the Stefan-Boltzmann constant, $\mathcal{E}$ is the emissivity of the solids, which was taken as 0.90 to be representative of soils [9], $A_{\text {Rad }}$ is the area of radiation heat transfer, which was equal to the minimum of either the total ash or the total shale surface area, $M$ is the extent of mixing between the materials. $\mathrm{M}$ at 100 assumes that all of the surface area is used for heat transfer, while $\mathrm{M}$ at 50 indicates that only half of the available surface area is used for heat exchange. This amount of radiation heat transfer is lost by the shale ash particles and gained by the oil shale particles.

During a specified time period, the total radiation heat transfer, $q_{r}$, was calculated using:

$$
q_{r}=Q_{r} \cdot \Delta t
$$

\section{Total heat transfer between granular materials}

Now the total heat transfer between materials is the sum of the convective and radiation heat transfer components. Thus:

$$
q_{i}=q_{n c, i}+q_{r, i} .
$$

However, the process being modelled is an unsteady state process, and thus the heat transfer results in changing temperatures of the materials. This heat transfer also drives some of the chemical reactions in the oil shale. The heat transfer is then recalculated at the next time step with the new material temperatures.

During the transient heat transfer, the specific enthalpy of the material, $\bar{H}_{i}(t)$, at time $t$ is calculated using:

$$
\bar{H}_{i}(t)=\bar{H}_{i}(t-1)+\frac{q_{i}(t)}{m_{i}},
$$

where $\bar{H}_{i}(t-1)$ is the specific enthalpy of the material prior to the heat transfer in the latest time step, $q_{i}(t)$ is the heat transfer in the last time step, $m_{i}$ is the original mass of that material type. The specific enthalpy was expressed per mass of original material. The thermodynamic model described above is used to calculate the new temperature of the materials due to the transient heat transfer. 


\section{Heat transfer scenarios}

To use this model, the sampling time needs to be small enough so that the heat transfer is approximated by a quasy-steady state conditions. A time step of 0.1 second was used in the following scenarios, and this resulted in an energy balance to within $1 \%$. A smaller sampling time would produce a better energy balance but would be more computationally intensive.

The following input is required to run a scenario in the developed model:

- ratio of oil shale to shale ash,

- sampling time to be used for the simulation,

- total running time of the simulation,

- extent of mixing between oil shale and shale ash,

- chemical composition, sensible heat and reaction parameters for both oil shale and shale ash,

- initial temperature of oil shale and shale ash, and

- particle size of the materials.

The model is used to determine the rate of heating of oil shale (and cooling of shale ash) under a range of conditions. The model is tested with four sets of scenarios, as shown in Table 2 . These scenarios vary the particle

Table 2. Heat transfer scenarios

\begin{tabular}{|c|c|c|c|c|c|}
\hline Scenario & $\begin{array}{c}\text { Particle } \\
\text { diameter, } \\
\mathrm{mm}\end{array}$ & $\begin{array}{c}\text { Material ratio } \\
\text { oil shale: } \\
\text { shale ash }\end{array}$ & $\begin{array}{c}\text { Initial oil shale } \\
\text { temperature, } \\
\text { K }\end{array}$ & $\begin{array}{c}\text { Initial shale } \\
\text { ash } \\
\text { temperature, } \mathrm{K}\end{array}$ & $\begin{array}{l}\text { Percent } \\
\text { mixed }\end{array}$ \\
\hline \multicolumn{6}{|c|}{ Particle diameter scenarios } \\
\hline D1 & 25 & $1: 3$ & 300 & 1100 & 50 \\
\hline D2 & 10 & $1: 3$ & 300 & 1100 & 50 \\
\hline D3 & 5 & $1: 3$ & 300 & 1100 & 50 \\
\hline D4 & 2 & $1: 3$ & 300 & 1100 & 50 \\
\hline D5 & 1 & $1: 3$ & 300 & 1100 & 50 \\
\hline \multicolumn{6}{|c|}{ Material ratio scenarios } \\
\hline $\mathrm{R} 1$ & 5 & $3: 1$ & 300 & 1100 & 50 \\
\hline $\mathrm{R} 2$ & 5 & 2:1 & 300 & 1100 & 50 \\
\hline R3 & 5 & 1:1 & 300 & 1100 & 50 \\
\hline $\mathrm{R} 4$ & 5 & $1: 2$ & 300 & 1100 & 50 \\
\hline R5 & 5 & $1: 3$ & 300 & 1100 & 50 \\
\hline R6 & 5 & $1: 4$ & 300 & 1100 & 50 \\
\hline \multicolumn{6}{|c|}{ Initial temperature of oil shale scenarios } \\
\hline T1 & 5 & $1: 3$ & 300 & 1100 & 50 \\
\hline $\mathrm{T} 2$ & 5 & $1: 3$ & 350 & 1100 & 50 \\
\hline $\mathrm{T} 3$ & 5 & $1: 3$ & 400 & 1100 & 50 \\
\hline $\mathrm{T} 4$ & 5 & $1: 3$ & 450 & 1100 & 50 \\
\hline \multicolumn{6}{|c|}{ Percent of mixing scenarios } \\
\hline M1 & 5 & $1: 3$ & 300 & 1100 & 10 \\
\hline M2 & 5 & $1: 3$ & 300 & 1100 & 25 \\
\hline M3 & 5 & $1: 3$ & 300 & 1100 & 50 \\
\hline M4 & 5 & $1: 3$ & 300 & 1100 & 75 \\
\hline M5 & 5 & $1: 3$ & 300 & 1100 & 100 \\
\hline
\end{tabular}


diameter, the material ratio, the initial temperature of the oil shale and the total extent of mixing between the materials.

The following figures show the transient response of the oil shale and shale ash materials. The dashed lines, which start at the higher temperature, represent the dynamic response of shale ash while the solid lines represent the dynamic heating of oil shale.

\section{Effect of reactions}

The following chart compares the dynamic response for a scenario where the chemical reactions of the oil shale have been disabled, compared to a scenario where the chemical reactions are activated.

The effect of the chemical reactions is observed for the oil shale as the roughly horizontal sections. Since these reactions are assumed to be irreversible (i.e. as you cool shale ash, the kerogen does not reform in the matrix) they are not observed during the cooling of the shale ash.

The inclusion of reactions also reduced the equilibrium temperature from $867 \mathrm{~K}$ for a system with no reactions to $697 \mathrm{~K}$ for a system with chemical reactions. This arises because the chemical reactions absorb heat at a uniform temperature. A typical example of this is the drying reaction, which occurs for the oil shale between $373 \mathrm{~K}$ and $413 \mathrm{~K}$, between 2 seconds and 10 seconds in Fig. 5. Further, the equilibrium temperature is reached quicker when chemical

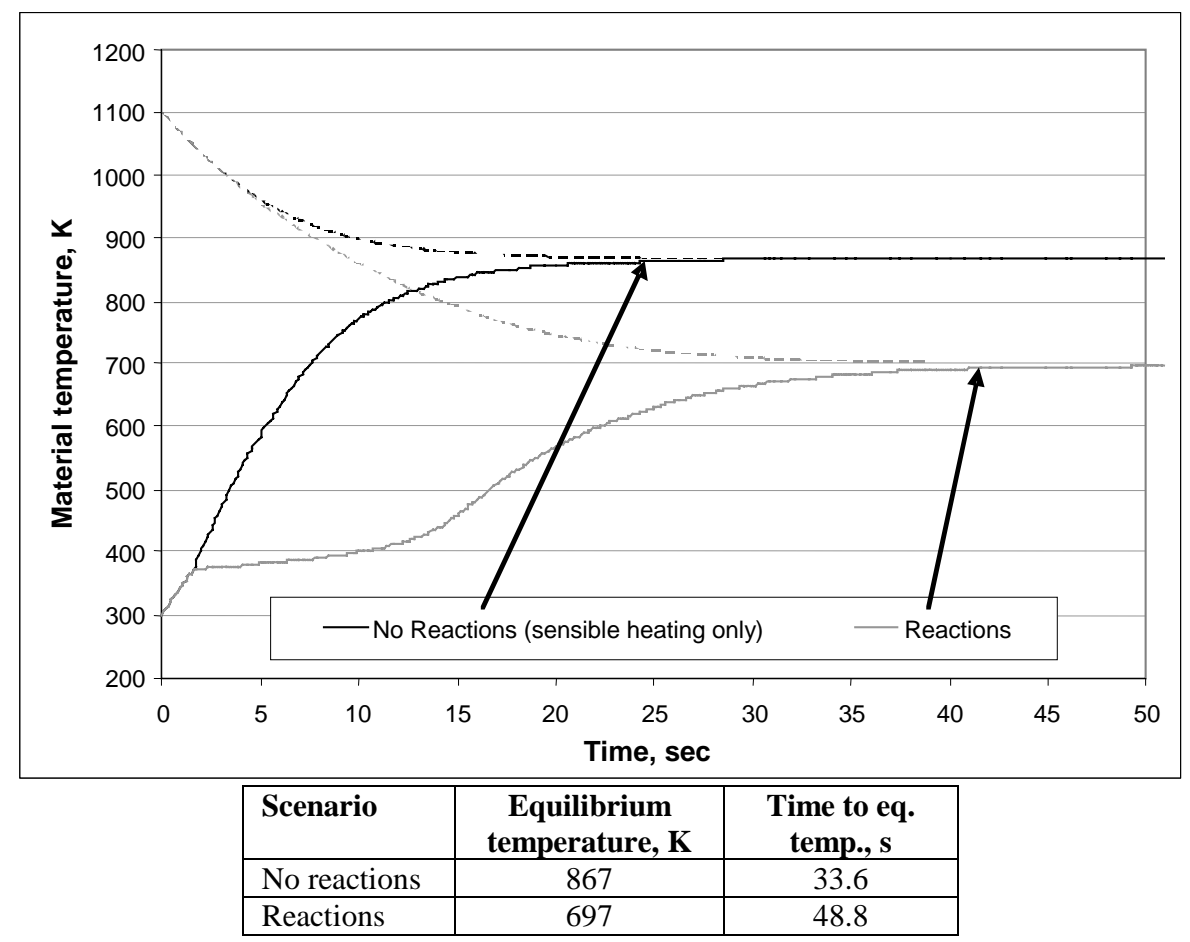

Fig. 5. The effect of removing chemical reactions to heat transfer dynamics. 
reactions are removed. Once again, chemical reactions for oil shale absorb heat while not significantly increasing the temperature of oil shale.

\section{Particle diameter scenarios}

The effects of changing the particle size diameters were simulated. The particle size of both oil shale and shale ash were assumed to be equal in each simulation.

As shown in Fig. 6, the same equilibrium temperature was reached for a wide range of particle sizes. This was expected since changing the particle size does not change the chemistry of the system or the overall energy balance between the materials. However, the time taken to reach this temperature is strongly dependent on the particle size. This reflects the smaller heat transfer area for a specified volume of larger particles compared to smaller particles. As described above, both the convective and radiation heat transfer are dependent on the external surface area of the oil shale and shale ash particles. Thus less heat transfer would occur between larger particles, which increases the time to reach the equilibrium temperature.

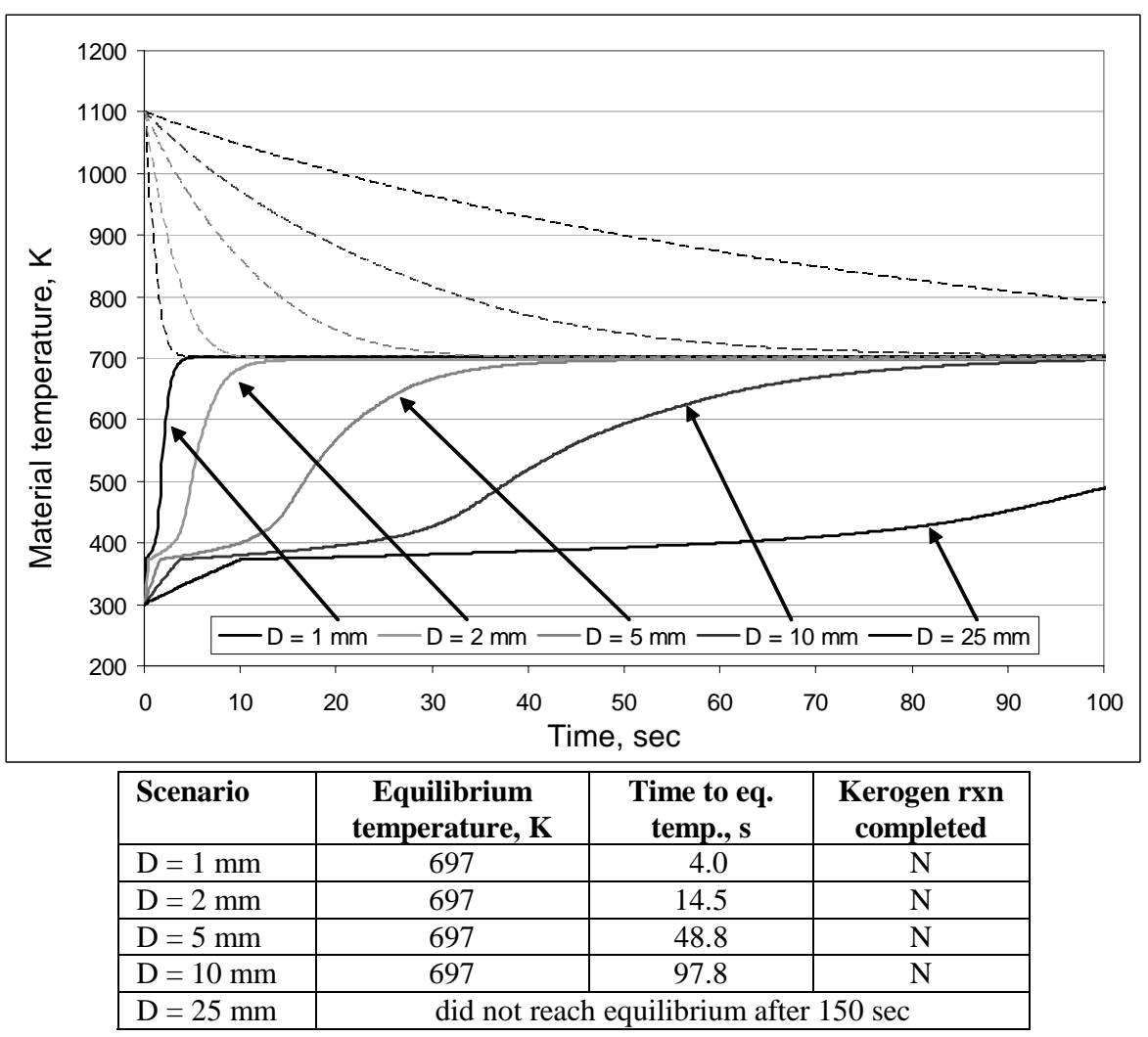

Fig. 6. The effect of particle size on the dynamic temperature profile. 


\section{Material ratio scenarios}

The ratio of oil shale to recycled shale ash is an operational variable for the Galoter and Alberta Taciuk processes. Figure 7 illustrates the dynamic temperature profile for ratios in the range of $3: 1$ to $1: 4$.

The final equilibrium temperature is strongly influenced by the material ratio. The equilibrium temperature approaches the shale ash initial temperature as more shale ash is added to the system, and conversely, the equilibrium temperature is closer to the initial oil shale temperature if large excesses of oil shale are used.

The dynamic response is interesting, in that the equilibrium temperature is reached quickest for a material ratio of 1:3. A longer time to equilibrium is observed for higher values of $\mathrm{R}$. This reflects the reduced heat transfer rate at the lower temperatures since the radiation heat transfer between the oil shale and shale ash particles become small at these lower temperature. Hence the dynamics are predominantly driven by convective heat transfer at these high values of $\mathrm{R}$.

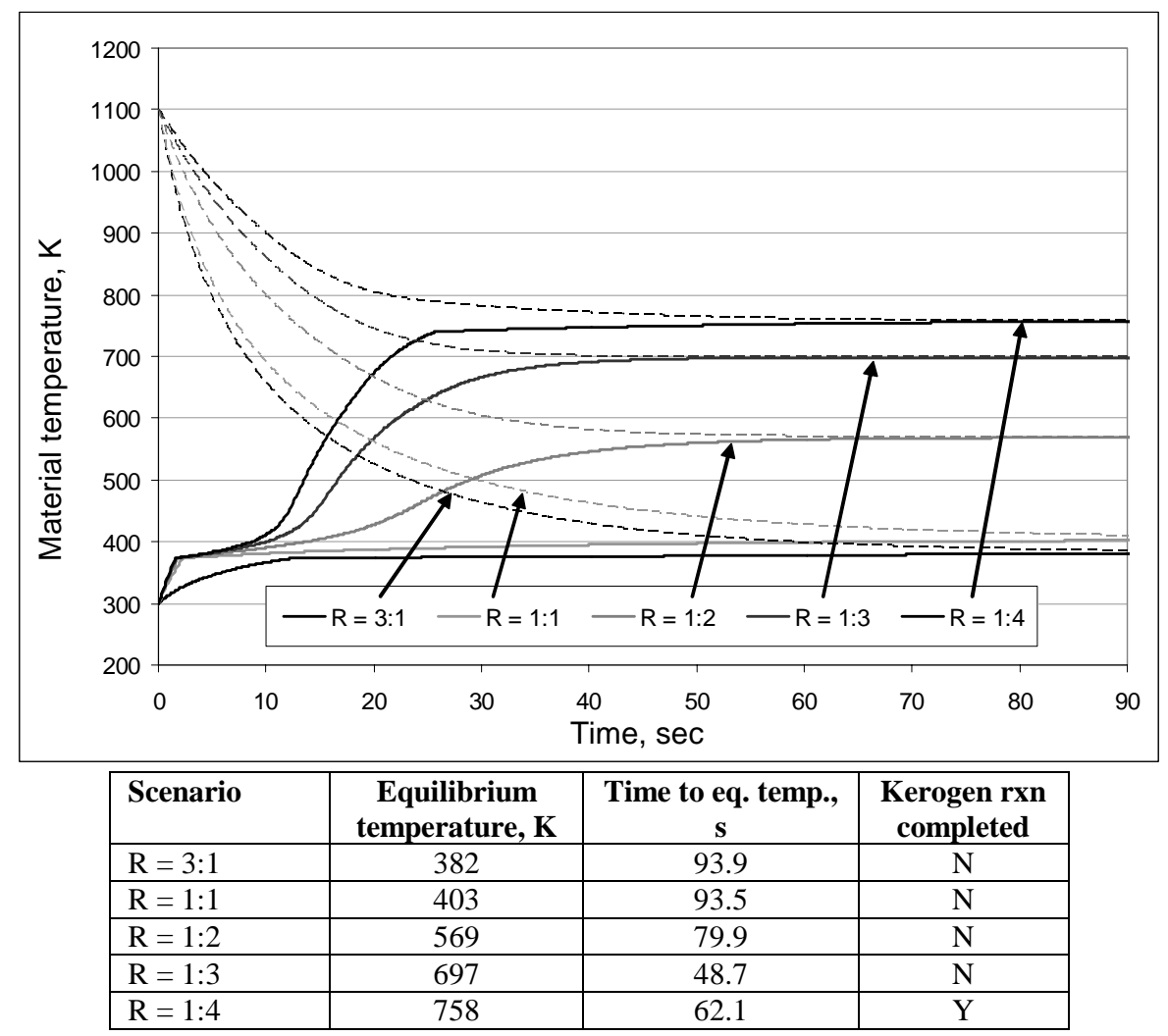

Fig. 7. The effect of material ratio on the dynamic temperature profile. 
However, the time taken to reach the equilibrium temperature for a lower ratio of 1:4 is also longer than that observed for the 1:3 ratio. This reflects that kerogen reactions for the oil shale under these conditions are completed, where they were not for the higher ratio of 1:3.

\section{Initial temperature of oil shale scenarios}

The initial temperature of the oil shale entering the retort is a controllable process parameter. If the temperature is too low, the kerogen reactions will not be completed while energy efficiency will be compromised if the temperature is too high. A range of temperatures from $300 \mathrm{~K}$ to $450 \mathrm{~K}$ was chosen to reflect oil shale at ambient conditions and dried oil shale.

The equilibrium temperature increases as the initial oil shale temperature increases. This is expected from a simple energy balance. The time taken to reach this equilibrium temperature decreases with increasing oil shale temperature. As shown in Fig. 8, this reflects that the oil shale reaches a higher temperature in the first 10 seconds since no energy is used to dry the oil shale. This results in a higher temperature system where radiation heat transfer becomes prominent.

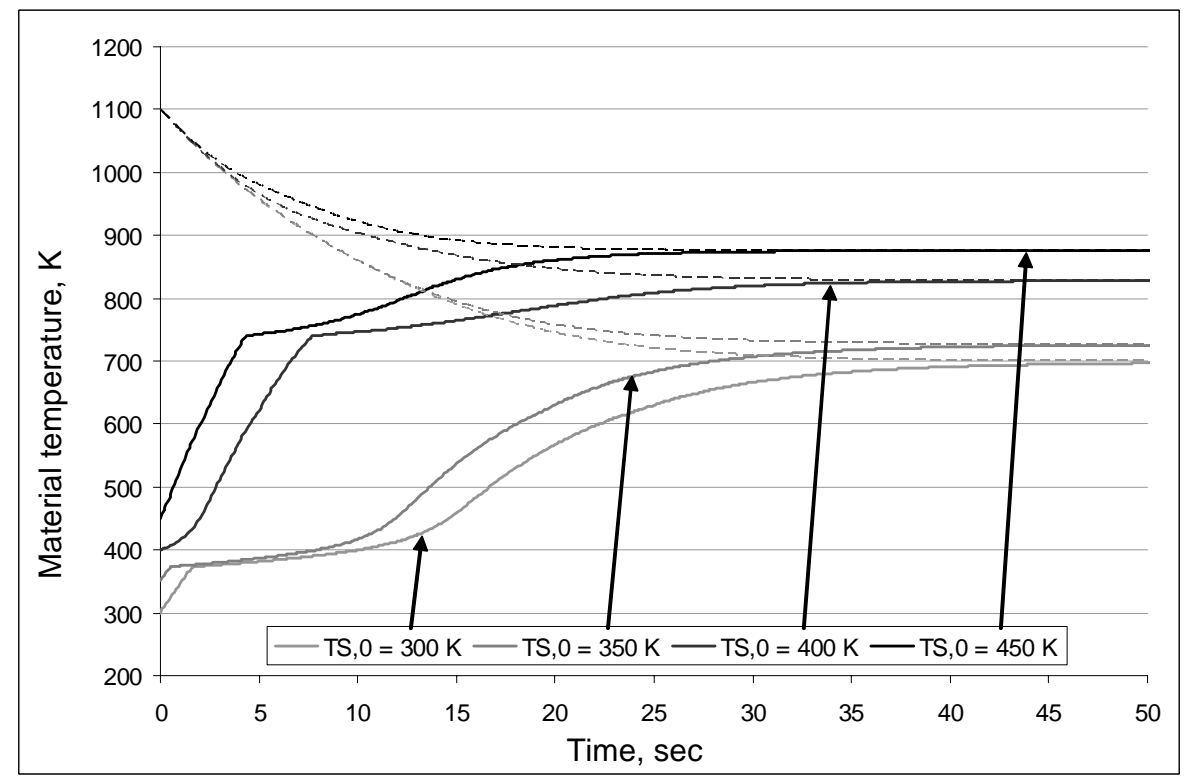

\begin{tabular}{|l|c|c|c|}
\hline Scenario & $\begin{array}{c}\text { Equilibrium } \\
\text { temperature, } \mathbf{K}\end{array}$ & $\begin{array}{c}\text { Time to eq. } \\
\text { temp., } \mathbf{s}\end{array}$ & $\begin{array}{c}\text { Kerogen rxn } \\
\text { completed }\end{array}$ \\
\hline $\mathrm{T}_{\mathrm{s}, 0}=300 \mathrm{~K}$ & 697 & 48.8 & $\mathrm{~N}$ \\
\hline $\mathrm{T}_{\mathrm{s}, 0}=350 \mathrm{~K}$ & 726 & 48.8 & $\mathrm{~N}$ \\
\hline $\mathrm{T}_{\mathrm{s}, 0}=400 \mathrm{~K}$ & 828 & 45.7 & $\mathrm{Y}$ \\
\hline $\mathrm{T}_{\mathrm{s}, 0}=450 \mathrm{~K}$ & 876 & 37.5 & $\mathrm{Y}$ \\
\hline
\end{tabular}

Fig. 8. The effect of the initial oil shale temperature on the dynamic temperature profile. 
The kerogen reaction was fully completed for the initial oil shale temperatures above $400 \mathrm{~K}$, but was partially completed for the scenario where the oil shale initial temperature was $350 \mathrm{~K}$.

\section{Percent mixed scenarios}

Radiation heat transfer between two different materials is driven by the "viewable" area between the two materials. The extent of mixing controls this. A range of scenarios were tested where the extent of mixing ranged from $10 \%$ to $100 \%$. The dynamic temperature profiles are shown in Fig. 9.

The equilibrium temperatures for these scenarios were the same, but the time taken to reach this temperature reduced with increased extent of mixing. In industrial applications, the extent of mixing is fixed in vertical retorts while the mixing increases with rotation in a rotary kiln retort.

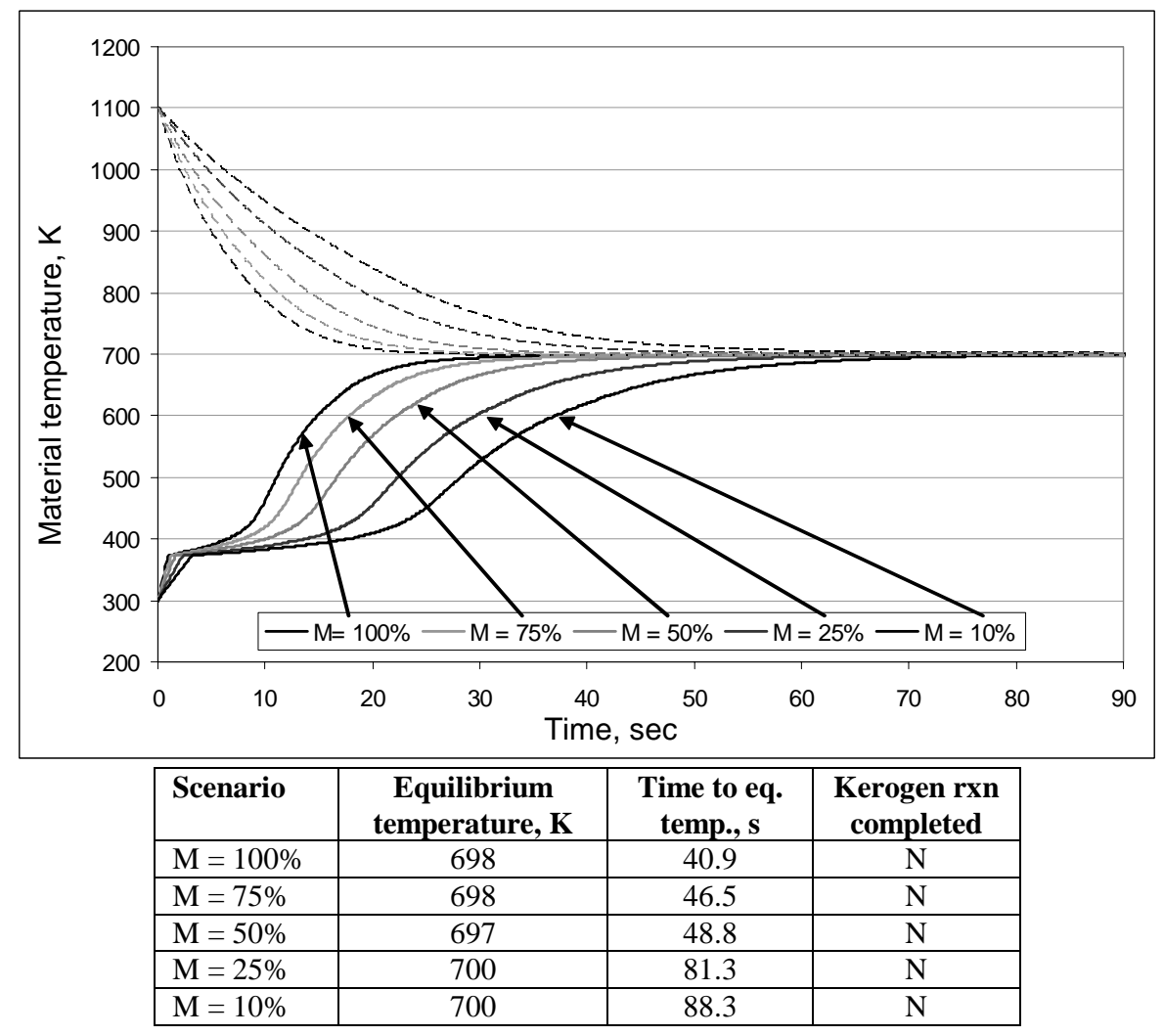

Fig. 9. The effect of mixing on the dynamic temperature profile. 


\section{Conclusions}

The model described in this paper illustrates granular medium heating, such as used in oil shale processes. While the model shown in this paper is for oil shale retorting, it can also be used on other granular medium applications, such as drying of foods.

The dynamic temperature response of a generic oil shale and shale ash are investigated for a range of scenarios. The scenarios illustrate the effects of particle diameter, material ratio, initial temperature and extent of mixing.

The equilibrium temperature is not dependent on the particle size nor the extent of mixing, however, both these variables do impact on the time taken to reach the equilibrium temperature. Hence reducing the particle size or increasing the amount of mixing would shorten the time to reach the equilibrium temperature. A balance must be sought to ensure that the process can still handle the smaller particle size and that the energy spent on size reduction or increased mixing is justified.

Changing the material ratio or initial temperature of oil shale also changes the equilibrium temperature and the time taken to reach this equilibrium temperature. For the generic oil shale selected in this paper, only the scenarios with either a material ratio less than 1:4 or the initial oil shale temperature greater than $400 \mathrm{~K}$ result in the completion of the kerogen retorting reactions. The range of scenarios provide sensible results, but confirming these results with experimental data remains a challenge since experimental measurement in this area remains difficult.

The model can be easily adjusted to estimate the dynamic thermal response for a specific oil shale. Future model developments include adding mixing dynamics and a particle size distribution.

\section{REFERENCES}

1. International Energy Agency. World Energy Outlook. - France, 2006.

2. Mölder, L. Estonian oil shale retorting industry at the crossroads // Oil Shale. 2004. Vol. 21, No. 2. P. 97-98.

3. Berkovich, A.J., Young, B. R., Levy, J.H. The application of modulated differential scanning calorimetry for determination of oil shale thermal properties during processing // Proceedings of the $25^{\text {th }}$ Australian and New Zealand Chemical Engineers' Conference and Exhibition, 29 September - 1 October 1997 / I. E. Aust (ed.). Rotorua, New Zealand, 1997.

4. Berkovich, A. J., Young, B. R., Ray, A., Schmidt, J. The effect of minerals on retorting enthalpies of some Australian tertiary oil shales // Fuel. 1998. Vol. 77, No. 9-10. P. 987-993.

5. Berkovich, A. J. The thermal characterisation of Australian Tertiary oil shales. Ph.D. dissertation, University of Technology, Sydney, Australia, 1999. 
6. Kok, M. V., Senguler, I., Hufnagel, H., Sonel, N. Thermal and geochemical investigation of Seyitomer oil shale// Thermochim. Acta. 2001. Vol. 371, No. 1-2. P. 111-119.

7. Yagi, S., Kunii, D. Studies on effective thermal conductivities in packed beds // AIChE. J. 1957. Vol. 3, No. 3. P. 373-381.

8. Kunii, D., Smith, J. M. Heat transfer characteristics of porous rocks // AIChE. J. 1960. Vol. 6, No. 1. P. 71-78.

9. Thomas, L. C. Heat Transfer. - Prentice Hall, New Jersey, USA, 1992.

10. Saatdjian, E., Large, J. F. Heat transfer in a countercurrent gas-solid, packed columns // J. Heat Transfer. 1988. Vol. 110, No. 2. P. 385-389.

Presented by A. Paist

Received May 7, 2007 